\title{
Meckel's diverticulum: Report of two cases and review of the literature
}

\author{
ERIC A. GOLDSMITH, DO \\ EDWARD A. GOTFRIED, DO
} 作 cations associated with this condition, intestinal obstruction is one of the most common. Rapid diagnosis and treatment are essential. Resection of the obstructing element is the treatment of choice. The authors report on their recent experience with two patients with Meckel's diverticulum, both of whom had intestinal obstruction as the presenting complication. The authors discuss the etiology and various complications of Meckel's diverticulum and present information on the frequency of associated mortality and morbidity.

The vitelline, or omphalomesenteric, duct is considered the most common diverticulum of the small intestine and, by some, the most common anomaly of the gastrointestinal tract. It was first described by Fabricius Hildamus in $1650 .^{1-4}$ Subsequent reports followed and ranged from Lavater in 1671 through Frautner in $1779.4,5$ However, it was Johann Friedrich Meckel, the younger, who, in 1808 , offered a comprehensive pathologic and embryologic description of the diverticular remnant that now bears his name. ${ }^{2,6}$

Forms of Meckel's diverticulum include inflammatory, obstructive, peptic with bleeding, and umbilical sinus. It can also occur as an intussusception. Patients require prompt surgical treatment and only at that time is the true etiology of the disease process discovered.

From Metropolitan Hospital, Springfield Divi $\cdots n$ Springfield, $\mathrm{Pa}$. At the time this article was writt $\mathrm{n}$, Dr Goldsmith was a second-year surgical resident; currently, he is attending general surgeon. Dr Gotfried is chief of surgery.

Reprint requests to Eric A. Goldsmith, DO, 4610 State Rd, Drexel Hill, PA 19026.
Our recent experience with Meckel's diverticulum consists of two cases with intestinal obstruction as the presenting complication.

\section{Report of cases}

\section{Case 1}

A 3-year-old boy had a 48-hour history of nausea and vomiting. The parents reported that the child also was experiencing colicky abdominal pains and had a temperature of $37.8^{\circ} \mathrm{C}$. The patient had not had a bowel movement for 2 days. The nausea and vomiting had started insidiously, occurring approximately 2 hours after eating. On presentation, the patient was anorexic. The patient had a twin sister who was in good health. There had been no prior medical illnesses nor was there any history of surgery. Birth of the twins had been vaginal and uncomplicated.

On physical examination the child appeared ill and lethargic and had dry mucous membranes. The pulse rate was 120 beats per minute, and the temperature was $38.6^{\circ} \mathrm{C}$. Examination of the abdomen revealed it to be tympanitic. Auscultation revealed hyperactive bowel sounds. There was a generalized tenderness with guarding to palpation.

Radiographic examination of the abdomen revealed distended small bowel loops with air fluid levels (Fig 1). Some air and feces were seen in the colon. The complete blood cell count (CBC) yielded a white blood cell count of $14,000 / \mu \mathrm{L}$ with 52 segmented cells, 14 bands, and 29 lymphocytes. Electrolyte, BUN, creatinine, and glucose levels were all normal. Urinalysis showed $3+$ ketones. The patient was admitted for fluid rehydration and observation.

The evening of admission, a repeat $\mathrm{CBC}$ yielded a total white blood cell count of $9300 / \mu \mathrm{L}$ with 44 segmented cells, 33 bands, and 23 lymphocytes. Diffuse abdominal pain continued, but positive rebound tenderness was now elicited in the right lower quadrant. The patient was taken to surgery with a diagnosis of acute appendicitis.

At surgery, purulent-free intraperitoneal fluid was discovered. The appendix was found to be 
mildly inflamed. Further exploration revealed an inflamed and suppurative Meckel's diverticulum encircling and entrapping a loop of small bowel. The tip of the diverticulum was attached to the umbilicus by a fibrous end remnant of the vitelline duct. The fibrous cord was cut, the Meckel's diverticulum was resected, and an incidental appendectomy was performed. The peritoneal fluid culture proved to be positive for Klebsiella pneumoniae and appropriate antibiotics were instituted. The patient had an uncomplicated postoperative course and was discharged on the sixth day following surgery.

\section{Case 2}

A 48-year-old woman was admitted for elective hysterectomy following a history of menometrorrhagia. Previous dilation and curettage had revealed adenomatous hyperplasia. The past medical history included hypertension. Past surgeries included a cholecystectomy and an appendectomy. Findings of a physical examination and results of laboratory studies were normal. An abdominal hysterectomy with bilateral salpingo-oophorectomy was performed.

On each of the first two postoperative days, the patient experienced several episodes of nausea with bilious vomiting. Physical examination showed the abdomen to be distended and there were present, but decreasing, bowel sounds. The patient experienced flatus and liquid stool. A nasogastric tube was inserted and bilious fluid was drained. The patient appeared to improve and the nasogastric tube was removed on the fifth postoperative day. That night and the next day the nausea and vomiting returned. Abdominal x-ray films, which originally suggested an adynamic ileus, now suggested a partial, mechanical small bowel obstruction (Fig 2). The decision was made to place a Cantor tube at this time. Conservative management continued for several days, and fluid and electrolyte abnormalities were corrected. Serial abdominal x-ray films revealed a slowly improving small bowel obstruction.

On the 13th postoperative day, the patient felt worse. Physical examination continued to demonstrate a mildly distended abdomen, but bowel sounds were now almost absent. Barium was placed through the Cantor tube. Serial x-ray films showed a nearly complete bowel obstruction. The white blood cell count was $9300 / \mu \mathrm{L}$ with 56 segmented cells, 16 bands, and 8 lymphocytes. The results of all remaining laboratory tests were normal. The patient had at no time been febrile. On the 14 postoperative day, the patient was taken to surgery.

At laparotomy, multiple adhesions were found

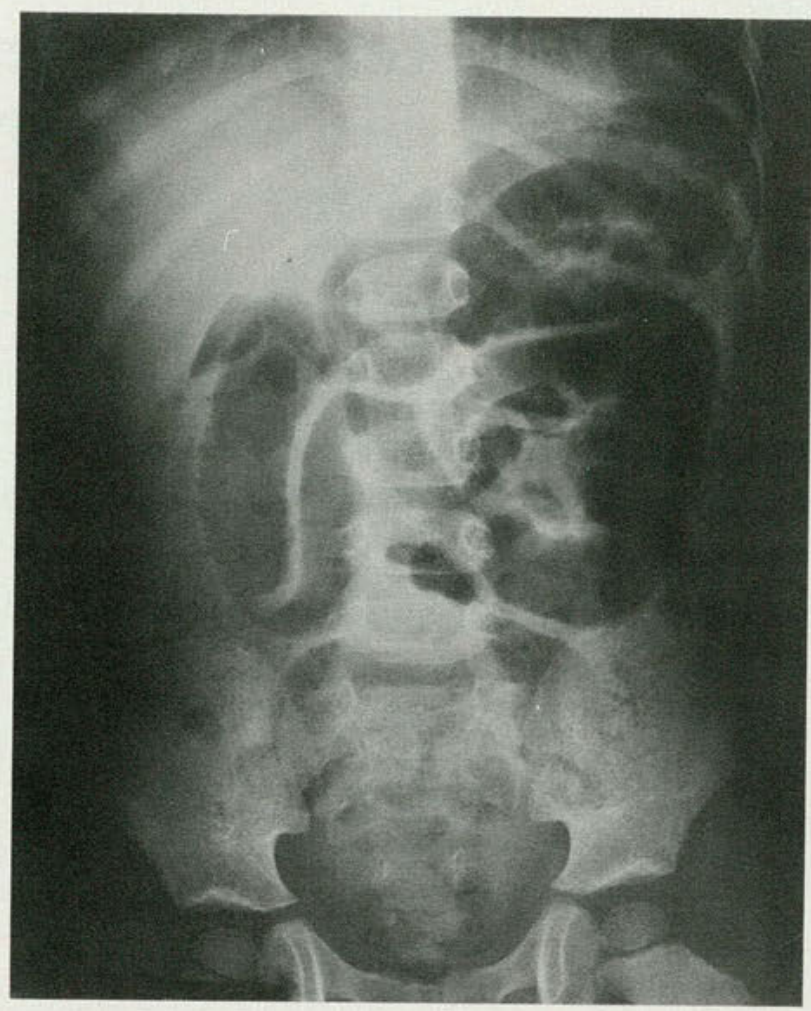

Figure 1. Abdominal x-ray film reveals distended small bowel loops with air fluid levels (case 1).

and lysed. Two loops of small intestine were found to be entrapped by a mesodiverticular band arising from a Meckel's diverticulum (closed-loop obstruction). The band was lysed and a diverticulectomy was performed.

After a prolonged hospital stay, the patient was discharged in satisfactory condition.

\section{Discussion}

From Meckel's description, the diverticulum is located on the antimesenteric border of the terminal ileum, usually $45 \mathrm{~cm}$ to $90 \mathrm{~cm}$ proximal to the ileocecal valve. It is a true diverticulum because it contains all intestinal layers. The diverticulum varies in both length and width, with length usually ranging from $1 \mathrm{~cm}$ to $12 \mathrm{~cm} .^{2,7,8}$

It is known today that Meckel's diverticulum represents one of the most frequent congenital anomalies and is reported to be found at $0.3 \%$ to $4 \%$ of all autopsies ${ }^{4,5,7}$ and at the time of $0.14 \%$ to $4.5 \%$ of abdominal surgeries. $1,7,9$ The ratio of occurrence in males to females is approximately 1.5 , approaching a 3.1 ratio for asymptomatic presentations. ${ }^{1,3,5,10,11}$ 
Meckel's diverticulum also is associated with other congenital malformations involving diverse organ systems. ${ }^{5,12}$ The majority of patients who have surgical complications of this lesion are 5 years of age or younger with a large percentage in the infant (2-year-old) group. ${ }^{10,13}$

The omphalomesenteric, or vitelline, duct represents the communication between the yolk sac and the developing midgut. Normally, this duct disappears between the fifth and ninth week of gestational age..$^{5,7,14}$ Of the paired vitelline vessels, the left disappears and the right remains to form the superior mesenteric artery. A small remnant of the right artery persists with its own mesentery to supply the Meckel's diverticulum. . ${ }^{7,10,11}$

The obliterative process begins at the umbilical end of the duct and extends toward the intestine.$^{9}$ Failure of complete obliteration of the vitelline duct results in the formation of anomalous structures. The type of structure is determined by the stage of arrest during the normal resorption of the duct. While Meckel's diverticulum accounts for up to $90 \%$ of all omphalomesenteric duct abnormalities, a variety of other types are also seen (Table 1). ${ }^{1,14}$

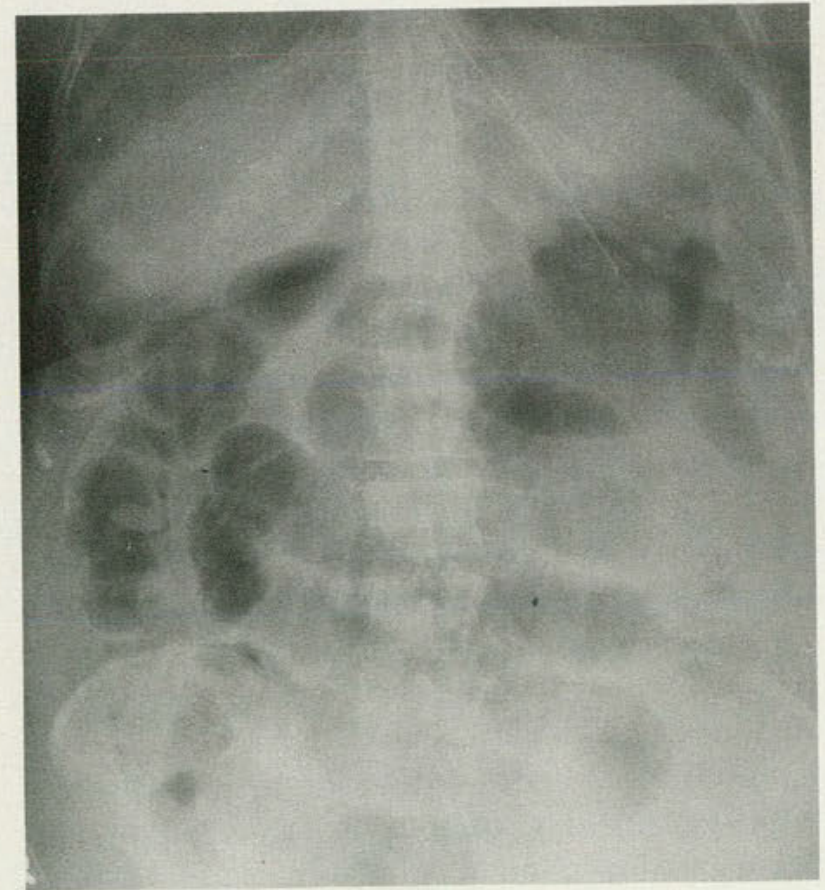

Figure 2. Abdominal $x$-ray film suggests a partial, mechanical small bowel obstruction (case 2).
Incomplete involution of the vitelline duct can result in a number of different anomalies, the most common of which is the Meckel's diverticulum. Because these vestigial structures may make their presence known in dramatic fashion, rapid diagnosis and treatment are essential. More than 50 years ago, Charles Mayo declared, "Meckel's diverticulum is frequently suspected, often looked for, and seldom found." 15

Because both the clinical and roentgenographic findings are usually nonspecific, the correct preoperative diagnosis is rarely made. Furthermore, few surgeons have extensive experience with the potentially catastrophic complications associated with this embryologic remnant. One reason may be the relative infrequency of the complications. Although about $2 \%$ of the general population have some form of this anomaly, only about $4.2 \%$ to $34 \%$ of affected persons actually have a complication develop..$^{3,5,12}$ Also, these complications may mimic other intra-abdominal emergencies, leading to delays in diagnosis and subsequent treatment.

Histologically, the omphalomesenteric duct contains totipotential cells. ${ }^{8}$ As a result, complications resulting from Meckel's diverticulum result not only from involution remnants but also from ectopic tissues.

Included in the ectopic tissues located in a Meckel's diverticulum are the commonly found gastric and pancreatic rests. ${ }^{1,5}$ The former tissue may give rise to peptic ulceration, usually at the base of the diverticulum or, at times, on the opposing ileal wall mucosa. ${ }^{9,10}$ Free perforation and gastrointestinal bleeding are resulting complications. In fact, when an otherwise thriving infant suddenly becomes pale and listless and exhibits usually painless melanotic or bloody stools, a bleeding Meckel's diverticulum should be suspected. ${ }^{7,9,16}$

Intussusception is caused by invagination of the diverticular base into the intestinal lumen. An abdominal mass is usually present on physical examination. Normal bowel movement may be seen before mucous and bloody stools appear. ${ }^{2,15}$

Littres hernia may involve entrapment of a Meckel's diverticulum in either a femoral 
Table 1

Causes of Intestinal Obstruction Secondary to Meckel's Diverticulum

- Fibrous band remnant with closed loop obstruction

- Intussusception

- Volvulus around a vitelline duct remnant

- Cicatricial narrowing of the small bowel

secondary to ulceration

- Prolapse of intestine through an umbilical fistula

- Incarceration of the diverticulum in hernia (Littre)

- Inflammation of the diverticulum with resulting obstruction

\section{Table 2}

Anomalies Resulting From Failure of Closure of the Vitelline Duct

- Meckel's diverticulum

- Umbilical fecal fistula

- Umbilical sinus

- Persistent fibrous cord

- Omphalomesenteric duct cyst

- Umbilical polyp

- Mesodiverticular vascular band

hernia ${ }^{2,15}$ or in an indirect inguinal hernia..$^{3,16}$ Umbilical polyps accompanied by excoriation of the adjacent skin should cause the physician to suspect a patent vitelline duct fistula, especially if simple cautery excision fails. ${ }^{2,8-9}$

Simple inflammation of a Meckel's diverticulum may occur, usually in the presence of a narrow-based diverticulum. ${ }^{8} \mathrm{~A}$ fecalith or foreign body entrapment may be the cause. Suppurative diverticulitis and abscess may result. ${ }^{2,3,10,15}$

Other less common complications involving Meckel's diverticulum include tuberculosis, regional enteritis, and a variety of tumors including carcinoid and adenocarcinoma tumors, as well as benign neoplasms. ${ }^{3,5,7}$

Of the possible complications associated with the vitelline duct remnant, intestinal obstruction is one of the most common. ${ }^{3-5,9,14,16}$ Here the classic symptoms of small bowel obstruction are present, including abdominal distention, pain, vomiting, and possibly a visible peristaltic wave on inspection of the abdomen. ${ }^{5}$
Other more diagnostic signs may include feculent discharge from the umbilicus or simply a fixed or indrawn umbilicus. ${ }^{9}$ Most patients are ill-appearing, are dehydrated, and have an electrolyte imbalance.

Radiographic studies have been used in an attempt to further aid in diagnosis. To this end, technetium scans, enhanced with pentagastrin and cimetidine, have been used with various degrees of success in the diagnosis of a bleeding Meckel's diverticulum. ${ }^{3,5}$ Some controversy surrounds the use of barium studies in suspected cases of small bowel obstruction. Antegrade barium studies take time, may not fill the diverticulum, and could potentially contaminate the intraperitoneal cavity if perforation is present. ${ }^{1,14}$ Barium enema can aid in excluding other diagnoses, but it confounds further abdominal x-ray films or scans. In the case of obstruction secondary to intussusception, the hydrostatic pressure generated by the enema has varied results of success in reducing the intussusception. ${ }^{10}$ However, astute use of the plain abdominal x-ray film can be most revealing, commonly indicating multiple dilated small-bowel loops. When acute obstruction has led to perforation, intraperitoneal ascites and possibly a soft tissue mass may be seen. Portal vein gas may also be seen and, although this is usually associated with necrotizing enterocolitis, any other cause of bowel necrosis-such as acute obstructioncan produce this finding. $1,5,14$

Intestinal obstruction secondary to vitelline duct remnants can occur in various ways (Table 2). Fibrous band attachment to the apex of the Meckel's diverticulum accounts for approximately $10 \%$ of omphalomesenteric duct abnormalities. ${ }^{1,14}$ This band can attach to virtually any place in the abdomen, especially the umbilicus. ${ }^{6,14}$ Failure of complete involution of either of the vitelline arteries gives rise to mesodiverticular vascular bands. ${ }^{6,10}$

The mechanisms of small bowel obstruction include closed-loop obstruction, direct band compression of the intestine, volvulus, intussusception, and entrapment in a mesodiverticular vascular band as in our second patient. Diverticular inflammation with localized reaction causing compression, angulation, and 
subsequent mechanical obstruction of the ileum is another mechanism ${ }^{6,11,13-15}$ and appears to be the cause of the intestinal obstruction found in our first patient. Interestingly, a band attachment of the apex of the Meckel's diverticulum to the umbilicus was also found in this patient, although this did not appear to be involved in the cause of the obstruction.

Resection of the obstructing element is the treatment of choice. This may be accomplished in several ways from simply cutting the obstructing cord to single or multiple segmental bowel resections. Most authors agree that, should a true diverticulum be present, diverticulectomy should be performed. Some argue that this is best accomplished by wedge resection with open transverse closure of the small bowel defect. ${ }^{2,15,16}$ The physician should bear in mind the practical importance of realizing that what appears as a simple band may be a vascular mesodiverticular remnant. Simply cutting this would result in dangerous bleeding because this band could retract and remain out of sight. ${ }^{10}$ Without question, a few hours of preoperative rehydration and correction of electrolyte imbalance are essential. Nasointestinal tube decompression is also suggested, although this should be tailored to the individual patient. ${ }^{13}$

Morbidity and mortality rates tend to be high, especially when intestinal obstruction is a complicating presentation of a vitelline duct remnant. Most series report their only mortalities from this particular complication. , $^{2,10,13}$ Mortality rates are also age dependent, with the rate increasing with the patient's youth. $2,4,9,10,12,13$ Under present conditions, we may expect an overall mortality rate of about $5 \%$. Morbidity rates are significant, especially in the presence of fistula, abscess, and postoperative obstruction secondary to adhesions. ${ }^{3,12}$

\section{Summary}

Meckel's diverticulum, one of the most common congenital abnormalities, can give rise to a variety of intra-abdominal complications, including ulceration, bleeding, intussusception, umbilical fistula, inflammation, and intestinal obstruction. Prompt diagnosis is essential because delay in treatment may lead to a life-threatening situation, especially in infants. Although the condition is usually regarded as a disease of the young, one of our patients was a middle-aged adult.

1. Dalinka M, Wunda J: Meckel's diverticulum and its compli cations, with emphasis on roentgenologic demonstrations. $D i$ agn Radiol 1973;106:295-298.

2. Mustard WT: Pediatric Surgery, ed 2. Chicago, Yearbook Medical Publishers, Inc, 1969, pp 864-868.

3. Mackey WC, Dineen P: A fifty-year experience with Meckel's diverticulum. Surg Gynecol Obstet 1983;156:56-64.

4. Haber JJ: Meckel's diverticulum: Review of literature and analytic study of twenty-three cases with particular emphasis on bowel obstruction. Am J Surg 1947;73:468-485.

5. Vane D, West K, Grosfield J: Vitelline duct anomalies: Experience with 217 childhood cases. Arch Surg 1987;122:542547.

6. Chance D, Hood R, Waugh J: Acute intestinal obstruction secondary to Meckel's diverticulum: Report of nine cases. AMA Arch Surg 1954;69:28-36.

7. DeBartolo H, Van Heerden J: Meckel's diverticulum. Ann Surg 1976;183:30-33.

8. Kiesewetter WB: Meckel's diverticulum in children. AMA Arch Surg 1957;75:914-919.

9. Aitken J: Remnants of the vitello-intestinal duct: A clinical analysis of 88 cases. Arch Dis Child 1953;28:393-398.

10. Rutherford R, Akers D: Meckel's diverticulum: A review of 148 pediatric patients, with special reference to the pattern of bleeding and to mesodiverticular vascular bands. Surgery 1966;59:618-626.

11. Moore T, Johnston AOB: Complications of Meckel's diverticulum. Br J Surg 1976;63:453-454.

12. Simms MH, Cockery JJ: Meckel's diverticulum: Its association with congenital malformation and the significance of atypical morphology. Br J Surg 1980;67:216-219.

13. Benson C, Linkner L: The surgical complications of Meckel's diverticulum in infants and children: An analysis of sixty cases. AMA Arch Surg 1956;73:393-398.

14. Gaisie G, Curnes JT, Scatliff JH, et al: Neonatal intestinal obstruction from omphalomesenteric duct remnants. AJR 1985;144:109-112.

15. Christensen E: Meckel's diverticulum as an abdominal emergency. Br Med J 1954; 41:1347-1350.

16. Eichenberger MR: Surgical conditions of the small intestine in infants and children, in Shackelford, RT (ed): Surgery of the Alimentary Tract. Philadelphia, WB Saunders Co, 1986, vol 10, pp 382-388. 